\title{
Analytic continuations of quantum cohomology
}

\author{
Yuan-Pin Lee, Hui-Wen Lin, and Chin-Lung Wang
}

\begin{abstract}
In the study of local model of ordinary flops, we introduce the Birkhoff factorizaton procedure to produce the generalized mirror map, which is the essential ingredient in performing analytic continuations of quantum cohomology in the Kähler moduli spaces. We survey this procedure in this article and provide an example to illustrate its validity.
\end{abstract}

\section{GW theory}

This article consists of the second author's talk at ICCM 2010 at Beijing under the same title, with more details filled in. The purpose is to introduce the results obtained by the authors in $[\mathbf{3}]$ and $[\mathbf{4}]$ and to work out a typical example.

Let $X$ be a projective manifold over $\mathbb{C}$ and $\bar{M}_{g, n}(X, \beta)$ be the moduli space of $n$-pointed stable maps $f:\left(C ; p_{1}, \ldots, p_{n}\right) \rightarrow X$ from a nodal curve $C$ with arithmetic genus $p_{a}(C)=g,[f(C)]=\beta \in N E(X)$, the Mori cone of effective one cycles.

Li-Tian and Behrend-Fantechi had constructed the virtual fundamental class $\left[\bar{M}_{g, n}(X, \beta)\right]^{\text {vir }} \in A_{D}\left(\bar{M}_{g, n}(X, \beta)\right) \otimes \mathbb{Q}$ with (virtual) dimension $D=\left(c_{1}(X) \cdot \beta\right)+$ $(1-g)(\operatorname{dim} X-3)+n$. And then the (descendent) Gromov-Witten invariants are defined by integration along these cycles: For $a_{i} \in H(X)$,

$$
\left\langle\tau_{k_{1}} a_{1}, \ldots, \tau_{k_{n}} a_{n}\right\rangle_{g, n, \beta}=\int_{\left[\bar{M}_{g, n}(X, \beta)\right]^{v i r}} \prod_{i=1}^{n} \psi_{i}^{k_{i}} e v_{i}^{*} a_{i},
$$

where $e v_{i}: \bar{M}_{g, n}(X, \beta) \rightarrow X$ is the evaluation morphism defined by $e v_{i}(f)=f\left(p_{i}\right)$ and the descendent insertions $\psi_{i}=c_{1}\left(L_{i}\right),\left.L_{i}\right|_{f}=T_{p_{i}}^{*} C$ is the class of the cotangent line bundle at the $i$-th marked section on the universal curve

$$
\bar{M}_{g, n+1}(X, \beta) \rightarrow \bar{M}_{g, n}(X, \beta) .
$$

The (primary) GW potential is the generating function of all GW invariants without descendent insertions $\left(k_{i}=0\right)$. Let $\left\{T_{i}\right\}$ be a cohomology basis of $H=$ $H(X),\left\{T^{i}\right\}$ be its dual basis, and $t=\sum t^{i} T_{i} \in H$ be a general element with $t^{i}$ being the coordinates. Let $\left\{q^{\beta}\right\}$ 's be the formal (Novikov) variables. Then

$$
F^{g}(t)=\sum_{n \geq 0, \beta \in N E(X)} \frac{q^{\beta}}{n !}\langle t, t, \cdots, t\rangle_{g, n, \beta} .
$$

2000 Mathematics Subject Classification. Primary 14N35, Secondary 14E30. 
The quantum product uses genus 0 theory with $n \geq 3$ marked points. Namely

$$
T_{i} *_{t} T_{j}=\sum_{k} \frac{\partial^{3} F^{0}(t)}{\partial t^{i} \partial t^{j} \partial t^{k}} T^{k}=\sum_{n, \beta, k} \frac{q^{\beta}}{n !}\left\langle T_{i}, T_{j}, T_{k}, t^{\otimes n}\right\rangle_{0,3+n, \beta} T^{k} .
$$

It is well known that the associativity of the quantum product is equivalent to the so called WDVV equations, which is also equivalent to the flatness of the Dubrovin connection

$$
\nabla_{i}^{z}=\frac{\partial}{\partial t^{i}}-\frac{1}{z} T_{i} *_{t}
$$

on the (trivial) tangent bundle $T H=H \times H \rightarrow H \ni t$, with $z \in \mathbb{C}^{\times}$being a free parameter.

In fact, the GW potential can be regraded as a function in two sets of variables. One is in the (complexified) Kähler moduli:

$$
\omega=B+i H \in \mathscr{K}_{X}^{\mathbb{C}}=H^{2}(X, \mathbb{R}) / H^{2}(X, \mathbb{Z})+i \mathscr{K}_{X},
$$

with $H$ being a Kähler class, such that

$$
q^{\beta}=e^{2 \pi i \int_{\beta} \omega}=e^{2 \pi i(B . \beta)} e^{-2 \pi(H . \beta)} .
$$

Conjecturally, $F^{g}(t)$ converges for $H$ large. At $\infty, q^{\beta}=0$ for $\beta \in N E(X) \backslash\{0\}$ and $*$ reduces to cup product. However this is still a wide opened conjecture. To avoid the convergence problem, one uses Novikov ring to work formally:

$$
N(X)=\mathbb{C}[\widehat{N E(X)}],
$$

the formal completion of the semigroup ring at the maximal ideal generated by all $q^{\beta}$ with $\beta \neq 0$.

The second set of variables are $t^{i}$ 's, organized as $t \in H$. For similar convergence reason one views $\left\{t^{i}\right\}$ as formal variables and $F^{g}(t)$ as formal power series. This later issue can be easily avoided since one can usually work with $n$-pointed GW invariants instead of the full generating functions.

It should also be remarked that $(Q H(X), *)=(H \otimes N(X),(), *,. \mathbf{1})$ is then a formal Frobenius manifold, where $($,$) is the Poincaré pairing and \mathbf{1}$ is the fundamental class. We will not need this notion in this note.

\section{Ordinary flops}

We are interested in analytic continuations in the $q^{\beta}$ variables arising from geometry. In order to make sense of this, we require that a second projective manifold $X^{\prime}$ to admit not just the same underlying vector spaces $H^{2}$ for the Mori cone to sit in but also the same space of insertions $t \in H$. The typical geometric situation leads to such an equivalence $H(X) \cong H\left(X^{\prime}\right)$ is given by the $K$-equivalence relation in birational geometry [6]. In this note we study the basic building block called the ordinary flops.

An ordinary $P^{r}$ flop $f: X \rightarrow X^{\prime}$ is a particular type of birational map between two projective manifolds such that it can be factorized through a pair of single blow-up and blow-down: $Y=\mathrm{Bl}_{Z} X=\mathrm{Bl}_{Z^{\prime}} X^{\prime}$, where $Z \subset X$ and $Z^{\prime} \subset X^{\prime}$ are the $f$-exceptional loci. More precisely, there is a commutative diagram of birational 
maps

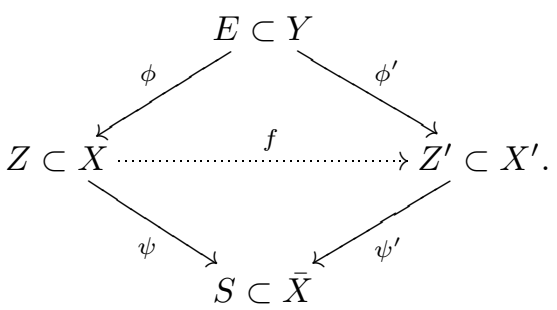

Here $\psi$ is the starting extremal contraction of flopping type, with its restriction to the exceptional loci $\bar{\psi}: Z=P_{S}(F) \rightarrow S$ being equipped with a projective bundle structure with $\operatorname{rank} F=r+1$. Also it is required that along each $\bar{\psi}$-fiber $\left(Z_{s} \cong P^{r}\right)$ one has $\left.N_{Z / X}\right|_{Z_{s}}=\mathscr{O}_{P^{r}}(-1)^{r+1}$. Then it is not hard to show that $f$ can be achieved by the blowing up map $\phi: Y \rightarrow X$ along $Z$, followed by the blowing down map $\phi^{\prime}: Y \rightarrow X^{\prime}$ along the second fiber direction of $E \rightarrow S$ (which is a $P^{r} \times P^{r}$ bundle). Moreover we have $Z^{\prime}=P_{S}\left(F^{\prime}\right)$ for another vector bundle $F^{\prime}$. Notice that $E=Z \times{ }_{S} Z^{\prime}$, and all the local structures are determined by

$$
N_{Z / X}=\bar{\psi}^{*} F^{\prime} \otimes \mathscr{O}_{Z}(-1), \quad N_{E / Y}=\mathscr{O}_{E}(-1,-1) .
$$

The graph closure of $f$ induces the canonical correspondence via the Künneth formula:

$$
\mathscr{F}=\left[\bar{\Gamma}_{f}\right] \in A\left(X \times X^{\prime}\right): H(X) \rightarrow H\left(X^{\prime}\right) .
$$

In fact it is equivalent to the map $\phi_{*}^{\prime} \circ \phi^{*}$.

It was shown in $[\mathbf{3}]$ that $\mathscr{F}$ gives equivalence of Chow motives and Poincaré pairing $g_{i j}=\left(T_{i} \cdot T_{j}\right)$, but NOT the cup product. Neither does $\mathscr{F}$ preserve the effectivity of curves. Indeed,

$$
\mathscr{F} \ell=-\ell^{\prime}
$$

with $\ell=\left[P^{1}\right] \in N E(X)$ the $\psi$-extremal ray and $\ell^{\prime}=\left[P^{1}\right] \in N E\left(X^{\prime}\right)$ the $\psi^{\prime}$ extremal ray. By the duality pairing between Kähler cone and Mori cone, this implies that under $\mathscr{F}: H(X) \cong H\left(X^{\prime}\right), \mathscr{K}_{X} \cap \mathscr{K}_{X^{\prime}}=\emptyset$. In particular, the comparison of $(Q H(X), *)$ and $\left(Q H\left(X^{\prime}\right), *\right)$ can make sense only under

$$
\mathscr{F} q^{\beta}=q^{\mathscr{F} \beta}
$$

and analytic continuations in the $q^{\ell}\left(=q^{-\ell^{\prime}}\right)$ variable. This is precisely the notion we intend to explore throughout this note.

A $P^{r}$ flop is called simple if the base is a point: $S=$ pt. This is the most studied case in the existing literature:

TheOREM 2.1 (Li-Ruan, $1997[5])$. For simple $P^{1}$ flops on 3-folds, which are also known as Atiyah flops, we have $\mathscr{F}: Q H(X) \stackrel{\sim}{\rightarrow} Q H\left(X^{\prime}\right)$.

ThEOREM 2.2 (LLW, $2006[3])$. The invariance of quantum ring, up to analytic continuations, holds true for simple $P^{r}$ flops in all dimensions.

Theorem 2.3 (Iwao-LLW, 2008 [2]). The invariance of full Gromov-Witten theory with $2 g+n \geq 3$ holds true for all simple $P^{r}$ flops.

In fact the invariance for the total ancestror potential in all genera is established in [2] based on results in [3]. Recently the authors studied the problem for general ordinary flops without the "simple" assumption [4] and obtained, among other things, the invariance of quantum rings under the split assumptions of the bundles 
$F$ and $F^{\prime}$. It is the main purpose of the following sections to present some typical examples to illustrate the general theory.

\section{Local models}

We consider the general case $S \neq$ pt. We will review certain background material in [4] without proof in order to state the final problem on local models. Using deformations to the normal cone for $Z \hookrightarrow X$ and $Z^{\prime} \hookrightarrow X^{\prime}$, ie. on the $X$ side we blow up the trivial family $X \times \mathbb{A}^{1}$ along $Z \times\{0\}$ :

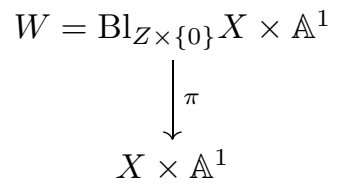

we get $W_{0}=Y \cup_{E} X_{l o c}$ and $W_{0}^{\prime}=Y \cup_{E} X_{l o c}^{\prime}$ above $0 \in \mathbb{A}^{1}$. Since both $X$ and $X^{\prime}$ share the same log pair $(Y, E)$, the natural goal is to reduce the problem on quantum invariance to the local model $f: X_{l o c} \rightarrow X_{l o c}^{\prime}$ where the projective local models are given by corresponding morphisms among projective bundles:

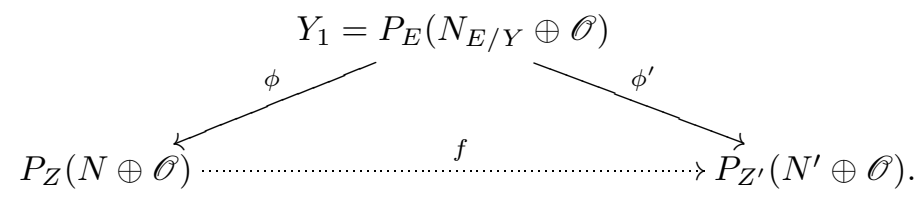

The reduction performed in [4] requires a two-steps degeneration analysis which, as in the simple case $[\mathbf{3}]$, is based on J. Li's algebraic version of the degeneration formula of GW invariants. However, we will not address on this reduction here. The outcome is that we may assume $X=X_{l o c}$, the double projective bundle

$$
\bar{p}: X \stackrel{p}{\rightarrow} Z \stackrel{\bar{\psi}}{\rightarrow} S
$$

with the price to pay that we need to deal with somewhat more general GW invariants called of $f$-special type.

Let $h=c_{1}\left(\mathscr{O}_{Z}(1)\right)$ and $\xi=c_{1}\left(\mathscr{O}_{X}(1)\right)=[E]$ be the relative hyperplane classes for $\bar{\psi}$ and $p$ respectively. By Leray-Hirsch,

$$
H(X)=H(S)[h, \xi] /\left(f_{F}(h), f_{N \oplus \mathscr{O}}(\xi)\right),
$$

where $f_{V}(x)$ denotes the Chern polynomial of the bundle $V$. Since $\operatorname{deg} f_{F}=r+1$ and $\operatorname{deg} f_{N \oplus \mathscr{O}}=r+2$, there is an obvious integral basis of $H(X)$ of the form

$$
\bar{t} h^{i} \xi^{j}, \quad \bar{t} \in H(S), \quad i \leq r, \quad j \leq r+1 .
$$

Lemma 3.1. $\mathscr{F}$ is $H(S)$-linear. It respects the product structure on the ideal generated by $\xi$, namely $\mathscr{F} \xi . \prod u_{i}=\xi^{\prime} \prod \mathscr{F} u_{i}$ and $\mathscr{F} h^{j}=\left(\xi^{\prime}-h^{\prime}\right)^{j}$ for $j \leq r$. In particular, $\mathscr{F}$ is a group isomorphism.

Now we discuss the Mori cone. We can always write $\beta \in N E(X)$ as $\beta=$ $d \ell+d_{2} \gamma+\left[\beta_{S}\right]$, where $\gamma$ is the $p$-fiber line class with $d_{2}=(\xi . \beta) . \beta_{S}=\bar{p}_{*} \beta$ with canonical lift $\left[\beta_{S}\right] \in N E(Z) \hookrightarrow N E(X)$ characterized by $\left(h \cdot\left[\beta_{S}\right]\right)=0$. There is a 
natural commutative diagram on one cycles:

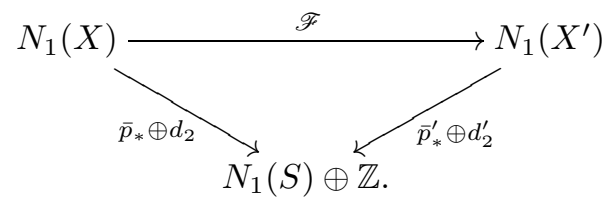

This reduces the analytic continuation problem to the one on sub fiber-series

$$
\langle\alpha\rangle_{g, \beta_{S}, d_{2}}=\sum_{d}\langle\alpha\rangle_{g, \beta_{S}, d_{2}, d} q^{d \ell}
$$

with $q^{\ell}$ being the only variable. From now on we consider only the case $g=0$ so we freely drop this index on genus henceforth.

We need to consider absolute local invariants of $f$-special type: Namely in the above multi insertions $\alpha$, we allow non-trivial descendent insertions $\tau_{j} a(j \neq 0)$ supported inside the isomorphic loci: $\left.a\right|_{Z}=0$. Equivalently, $\xi \mid a$.

\section{Analytic continuations}

There are four major steps toward the analytic continuations on local models. Namely (1) quantum corrections by extremal rays, (2) reduction by the dvisorial reconstruction to the quasi-linearity, (3) Birkoff factorizations (BF) and the generalized mirror transformations (GMT), and the final step (4) analytic continuations of BF/GMT. The first two steps are precisely the steps needed in the simple case $[\mathbf{3}]$. It is generalized to the non-simple case in [4], though technically much more involved due to the presence of bundles $F$ and $F^{\prime}$. Steps (3) and (4) are new for the non-simple case, which is also the main theme of this note.

Step 1: Extremal series $\left(\beta_{S}, d_{2}\right)=\overrightarrow{0}$. We only review the case $S=$ pt in $[\mathbf{3}]$ to fix the ideas. The general case holds with more complicate formulas involving $c_{i}=c_{i}(F)$ and $c_{i}^{\prime}=c_{i}\left(F^{\prime}\right)$.

First of all, consider 3 classes $a_{i} \in A^{k_{i}}(X)$ with $1 \leq k_{i} \leq r, k_{1}+k_{2}+k_{3}=$ $\operatorname{dim} X=2 r+1$. Then the defect of the cup product is given by

$$
\begin{aligned}
& \left(\mathscr{F} a_{1} \cdot \mathscr{F} a_{2} \cdot \mathscr{F} a_{3}\right)^{X^{\prime}}-\left(a_{1} \cdot a_{2} \cdot a_{3}\right)^{X} \\
& \quad=(-1)^{r}\left(a_{1} \cdot h^{r-k_{1}}\right)^{X}\left(a_{2} \cdot h^{r-k_{2}}\right)^{X}\left(a_{3} \cdot h^{r-k_{3}}\right)^{X} .
\end{aligned}
$$

Secondly, let $a_{i} \in A^{k_{i}}(X)$ with $\sum k_{i}=2 r+1+(n-3)$. Using localization and classical mirror symmetry techniques (plus reconstruction to be stated below), one proves the generalized multiple cover formula:

$$
\begin{aligned}
& \left\langle a_{1}, \ldots, a_{n}\right\rangle_{n, d \ell}^{X} \\
& \quad=(-1)^{(r+1)(d-1)} N_{k_{1}, \ldots, k_{n}} d^{n-3}\left(a_{1} \cdot h^{r-k_{1}}\right)^{X} \cdots\left(a_{n} \cdot h^{r-k_{n}}\right)^{X} .
\end{aligned}
$$

To see this leads to quantum corrections to the cup product, consider the rational form of the geometric series

$$
\mathbf{f}(q):=\frac{q}{1-(-1)^{r+1} q}=\sum_{d \geq 1}(-1)^{(r+1)(d-1)} q^{d} .
$$

It satisfies the functional equation $E(q):=\mathbf{f}(q)+\mathbf{f}\left(q^{-1}\right)+(-1)^{r+1}=0(E(q)$ is the formal Euler series), which will be the main source of analytic continuations. 
Indeed, for extremal functions $\langle\alpha\rangle_{0}:=\sum_{d=0}^{\infty}\langle\alpha\rangle_{d \ell} q^{d \ell}$,

$$
\begin{aligned}
& \left\langle\mathscr{F} a_{1}, \mathscr{F} a_{2}, \mathscr{F} a_{3}\right\rangle_{0}^{X^{\prime}}-\mathscr{F}\left\langle a_{1}, a_{2}, a_{3}\right\rangle_{0}^{X} \\
& \quad=\left(a_{1} \cdot h^{r-k_{1}}\right)\left(a_{2} \cdot h^{r-k_{2}}\right)\left(a_{3} \cdot h^{r-k_{3}}\right)\left((-1)^{r}-\mathbf{f}\left(q^{\ell^{\prime}}\right)-\mathbf{f}\left(q^{-\ell^{\prime}}\right)\right)=0 .
\end{aligned}
$$

For $n \geq 4$, let $\delta=q^{\ell} \partial / \partial q^{\ell}=-q^{\ell^{\prime}} \partial / \partial q^{\ell^{\prime}}$ be the power operator. Then

$$
\begin{aligned}
\langle\mathscr{F} & \left.a_{1}, \cdots, \mathscr{F} a_{n}\right\rangle_{0}^{X^{\prime}}-\mathscr{F}\left\langle a_{1}, \cdots, a_{n}\right\rangle_{0}^{X} \\
& =(-1)^{n} N_{k_{1}, \ldots, k_{n}} \prod_{i=1}^{n}\left(a_{i} . h^{r-k_{i}}\right) \delta^{n-3}\left(\mathbf{f}\left(q^{\ell^{\prime}}\right)+\mathbf{f}\left(q^{-\ell^{\prime}}\right)\right)=0 .
\end{aligned}
$$

These explains the invariance of quantum product restricted to the extremal rays.

Step 2: Now we come back to general $S$ and consider the $\mathscr{F}$-invariance of

$$
\left\langle\tau_{k_{1}} t_{1} \xi^{j_{1}} h^{l_{1}}, \cdots, \tau_{k_{n}} t_{n} \xi^{j_{n}} h^{l_{n}}\right\rangle_{\beta_{S}, d_{2}}^{X}
$$

up to analytic continuations, for all $\left(\beta_{S}, d_{2}\right) \neq(0,0)$, where $t_{i} \in H(S)$ and $k_{i} \neq 0$ only if $j_{i} \neq 0$ (invariants of $f$-special type).

The divisorial reconstruction theorem of Lee-Pandharipande says that

$$
\begin{aligned}
& e_{i}^{*} L \cap\left[\bar{M}_{0, n}(X, \beta)\right]^{\text {vir }} \\
& =\left(e_{j}^{*} L+(\beta . L) \psi_{j}\right) \cap\left[\bar{M}_{0, n}(X, \beta)\right]^{\text {vir }}-\sum_{\beta_{1}+\beta_{2}=\beta}\left(\beta_{1} . L\right)\left[D_{i, \beta_{1} \mid j, \beta_{2}}\right]^{\text {vir }} .
\end{aligned}
$$

In principle this allows us to move each divisor factor like $\xi$ and $h$ to the last point. For the invariance property to be compatible with the process, it is crucial to make sure that the last boundary splitting terms do not lead to 2-point GW extremal series since it is not $\mathscr{F}$-invariant.

If $\vec{j} \neq 0$, it is reduced to quasi-linearity: That is, $\mathscr{F}$-invariance of

$$
\left\langle t_{1}, \ldots, t_{n-1}, \tau_{k} a . \xi\right\rangle_{\beta_{S}, d_{2}}^{X} .
$$

If $\vec{j}=0$ and $d_{2} \neq 0$, one may insert $\xi$ by hand based on the divisor equation. For $d_{2}=0$, however, we arrived at a new case which has no counterpart in the simple case. The actual process in $[4]$ is rather sophisticated. It is based on a clever use of the WDVV equations to create a triangular system of linear equations. By solving this system we again reduce to the case with non-trivial appearance of $\xi$, and thus to the quasi-linearity conjecture. We remark that so far everything works for general bundles $F$ and $F^{\prime}$ without the split assumption.

\section{BF and GMT}

Let $\tau=\sum_{i} \tau^{i} T_{i}=\tau_{1}+\tau_{2} \in H, \tau_{1} \in H^{2}$. The $J$-function is the generating function of 1-descendent invariants:

$$
\begin{aligned}
J^{X}\left(\tau ; z^{-1}\right) & =1+\frac{\tau}{z}+\sum_{\beta, n, i} \frac{q^{\beta}}{n !} T^{i}\left\langle\frac{T_{i}}{z(z-\psi)}, \tau^{\otimes n}\right\rangle_{0, n+1, \beta} \\
& =e^{\frac{\tau}{z}}+\sum_{\beta \neq 0, n, i} \frac{q^{\beta}}{n !} e^{\frac{\tau_{1}}{z}+\left(\tau_{1} \cdot \beta\right)} T^{i}\left\langle\frac{T_{i}}{z(z-\psi)}, \tau_{2}^{\otimes n}\right\rangle_{0, n+1, \beta} .
\end{aligned}
$$

For the second equality we have to employ the deliton equation and divisor equation (with descendent) to take out the relevant cohomology classes from the bracket. 
Being a generating function, it is perhaps not too surprise that $J$ also gives rise to fundamental solutions (flat frame) of the Dubrovin connection:

$$
z \frac{\partial}{\partial t^{i}} z \frac{\partial}{\partial t^{j}} J(t)=\sum_{k} c_{i j}^{k}(t) z \frac{\partial}{\partial t^{k}} J(t)
$$

with $c_{i j}^{k}(t)=\sum_{m} F_{i j m}^{0}(t) g^{m k}$ being the structure constants of $*_{t}$. (Here $g_{m k}=$ $\left(T_{m}, T_{k}\right)$ is the Poincaré pairing and $\left(g^{m k}\right)$ is the inverse matrix.)

Notice that for a flop $f: X \rightarrow X^{\prime}$, in general we can not expect $J^{X}(t)$, as well as the frame $\partial_{j} J^{X}(t)$, to be $\mathscr{F}$-invariant. However it is precisely our plan to show that the coefficients $c_{i j}^{k}(t)$ 's are $\mathscr{F}$-invariant. This is somewhat similar to, though much more advanced than, the Frenet frame in the classical curve theory in Euclidean spaces.

The real difficulty in dealing with the (big) $J$ function lies in the fact that in general we do not have direct control of all the $\partial_{j}$ directions. In practical situations if we are dealing with projective manifold $X$ equipped with $\mathbb{C}^{\times}$actions, localizations on the stable map moduli spaces leads to certain combinatorial data related to the $J$ function in some partial directions. Special efforts then need to be made in each particular case to extract the relevant partial information on $J$ determined by these localization data.

For this reason, we now assume that $f$ is split: Namely

$$
F=\bigoplus_{i=0}^{r} L_{i}, \quad F^{\prime}=\bigoplus_{i=0}^{r} L_{i}^{\prime}
$$

for some line bundles $L_{i}$ 's and $L_{i}^{\prime}$ 's on $S$. The Chern polynomials take the form

$$
f_{F}=\prod a_{i}=\prod\left(h+L_{i}\right), \quad f_{N \oplus \mathscr{O}}=b_{r+1} \prod b_{i}=\xi \prod\left(\xi-h+L_{i}^{\prime}\right) .
$$

Step 3: Birkhoff factorization (BF). For $\beta=\beta_{S}+d \ell+d_{2} \gamma$, we denote by $\mu_{i}:=\left(L_{i} \cdot \beta_{S}\right), \mu_{i}^{\prime}:=d_{2}+\left(L_{i}^{\prime} \cdot \beta_{S}\right)$, and then

$$
\left(a_{i} \cdot \beta\right)=d+\mu_{i}, \quad\left(b_{i} \cdot \beta\right)=-d+\mu_{i}^{\prime} .
$$

From fiber-wise $\mathbb{C}^{\times}$localization, it is natural to consider the following product of Gamma factors (we set $\lambda_{\beta}=\left(c_{1}(X / S) \cdot \beta\right)$ ):

$$
I_{\beta}^{X / S}:=\frac{1}{z^{\lambda_{\beta}}} \frac{\Gamma\left(1+\frac{\xi}{z}\right)}{\Gamma\left(1+\frac{\xi}{z}+d_{2}\right)} \prod_{i=0}^{r} \frac{\Gamma\left(1+\frac{a_{i}}{z}\right)}{\Gamma\left(1+\frac{a_{i}}{z}+\mu_{i}+d\right)} \frac{\Gamma\left(1+\frac{b_{i}}{z}\right)}{\Gamma\left(1+\frac{b_{i}}{z}+\mu_{i}^{\prime}-d\right)} .
$$

The hypergeometric modification of the base $J$ function $J^{S}(\bar{t})$, modified by the fiber information of the split toric bundle $\bar{p}: X \rightarrow S$, is defined to be

$$
I\left(D, \bar{t} ; z, z^{-1}\right)=\sum_{\beta \in N E(X)} q^{\beta} e^{\frac{D}{z}+(D \cdot \beta)} I_{\beta}^{X / S} J_{\beta_{S}}^{S}(\bar{t}),
$$

where the variable $D=t^{1} h+t^{2} \xi$ is the fiber divisor and $\bar{t} \in H(S)$. The reason for this extra exponential factor comes from the fact that the relative factor $I^{X / S}$ takes care only the (small) divisor variables in the GW theory.

The difference between $J$ and $I$ is that the former is a power series in $z^{-1}$ while the later is only a Laurent series in $z^{-1}$, as it generally contains positive $z$ powers 
when $\lambda_{\beta}$ is sufficiently negative. A result of J. Brown [1] shows that $I$ lies in the "Lagrangian cone" spanned by $J$. In practical terms this means that

$$
I\left(D, \bar{t} ; z, z^{-1}\right) \in N(X)[z]\left[z \partial_{\tau^{0}}, \cdots, z \partial_{\tau^{N}}\right] J(\tau),
$$

where $\left\{\tau^{i}\right\}$ is a complete set of dual coordinates on $H$.

Since the relation between the variables $\hat{t}=(D, \bar{t})$ and $\tau$ is not clear in the above form, in order to put it into use we prefer to switch the differential operators to the LHS. This cannot be done näively since we do not have all the directions (variables) available on the $I$ factor. Indeed we can only make use of $t^{i}, i=1,2$ in the fiber directions. It turns out that this is enough for our purpose since we can perform higher derivatives:

THEOREM 5.1 (BF/GMT [4]). There is a unique differential operator

$$
B(z)=1+\sum q^{\beta} c_{\beta}\left(z, z \partial_{t^{i}}, z \partial_{\bar{t}^{s}}\right), \quad i=1,2,
$$

such that

$$
J\left(\tau ; z^{-1}\right)=B(z) I\left(\hat{t} ; z, z^{-1}\right)=1+O(1 / z) .
$$

The generalized mirror transformation is then determined by $\tau(\hat{t})=\hat{t}+$ $\sum q^{\beta} g_{\beta}(\hat{t})$ which is simply the $1 / z$ coefficient of the $R H S$.

We indicate how to compute $B(z)$ inductively. By induction on $\beta \in N E(X)$ we may construct $c_{\beta}$ by removing the top $z$ power

$$
A=z^{k} q^{\beta}\left(\bar{T}_{s} h^{i} \xi^{j}\right), \quad i \leq r,
$$

in $I_{\beta} q^{\beta}$ via näive "quantization":

$$
I-\hat{A} I=I-z^{k} q^{\beta}\left(z \partial_{\bar{t}^{s}}\right)\left(z \partial_{t^{1}}\right)^{i}\left(z \partial_{t^{2}}\right)^{j} I .
$$

The target term is removed since

$$
\hat{A} I_{\beta=0}=A \text {. }
$$

Also all the newly added terms are of higher order in $N E(X)$. Then we keep on removing all the lower $z^{\geq 0}$ terms.

Reduction to proving $\mathscr{F}$-invariance of BF/GMT.

We start by noticing the symmetry of roots (Chern monomials):

$$
\mathscr{F} a_{i}=\mathscr{F}\left(h+L_{i}\right)=\left(\xi^{\prime}-h^{\prime}+L_{i}\right)=b_{i}^{\prime}, \quad \mathscr{F} b_{i}=a_{i}^{\prime} .
$$

To consider the similar notion for effective one cycles, we call a class $\beta \in N E(X)$ being $\mathscr{F}$-effective if $\mathscr{F} \beta \in N E\left(X^{\prime}\right)$ too. It is not hard to show that

$$
\begin{aligned}
\beta \in N E(X) & \Longleftrightarrow \exists i,\left(\beta \cdot a_{i}\right)=d+\mu_{i} \geq 0, \\
\mathscr{F} \beta \in N E\left(X^{\prime}\right) & \Longleftrightarrow \exists i,\left(\beta \cdot a_{i}^{\prime}\right)=-d+\mu_{i}^{\prime} \geq 0 .
\end{aligned}
$$

If $\beta$ is NOT $\mathscr{F}$-effective, then by using $\Gamma(1+x)=x \Gamma(x)$, one calculates easily that (notice that $\xi=b_{r+1}$ and $\prod_{i=0}^{r+1} b_{i}=0$ )

$$
I^{X / S} \cdot \xi=\prod(\cdots) \prod_{i=0}^{r} \frac{b_{i}}{z} \cdot \xi=0 .
$$

If $\beta$ is $\mathscr{F}$-effective, by symmetry of roots, we find

$$
\mathscr{F} I^{X} \cdot \xi=I^{X^{\prime}} \cdot \xi^{\prime}
$$

Finally, since

$$
J^{X}(\tau(\hat{t})) \cdot \xi=B(z) I^{X}(\hat{t}) \cdot \xi
$$


by another induction on the number of insertions we need only to show the $\mathscr{F}$ invariance for $B(z)$ and $\tau(\hat{t})$ in order to prove the quasi-linearity.

This last task is the final step (Step 4) of the procedure. The underlying principle for us to believe this to be true is because that $I^{X}$ is a solution to the Picard-Fuchs equation

$$
P F=\prod z \partial_{a_{j}}-q^{\ell} \prod z \partial_{b_{j}}
$$

as one may check easily. (Here we abuse the notation by writing $\partial_{v}$ to denote the directional derivative in $v$. Thus if $v=\sum v^{i} T_{i} \in H^{2}$ then $\partial_{v}=\sum v_{i} \partial_{t^{i}}$.) It happens that

$$
\mathscr{F} P F^{X}=-q^{-\ell^{\prime}} P F^{X^{\prime}}
$$

which simply means that both $I^{X}$ and $I^{X^{\prime}}$ satisfy the same Picard-Fuchs equation, but in different coordinate charts " $\left|q^{\ell}\right|<1$ " and " $\left|q^{\ell}\right|>1$ " respectively.

This is the starting point toward the proof in [4]. In the following section we will work out directly an example to illustrate the general principle.

\section{A typical example: BF}

Let $f: X \rightarrow X^{\prime}$ be a local $P^{1}$ flop over $S=P^{1}$ of type

$$
\left(S, F, F^{\prime}\right)=\left(P^{1}, \mathscr{O}(-5) \oplus \mathscr{O}, \mathscr{O}(1) \oplus \mathscr{O}\right) .
$$

Hence $a_{0}=h-5 p, a_{1}=h, b_{0}=\xi-h+p$ and $b_{1}=\xi-h$.

Let $d_{2}=0$. The case with $d_{2}>0$ is much easier since the highest possible $z$ power decreases in $d_{2}$. So $\beta=d \ell+s[S]$. Denote by $q:=q^{\ell}, \bar{q}:=q^{[S]}$.

Since $H\left(P^{1}\right)=\mathbb{Z}[p] /\left(p^{2}\right)$, in order to work out the explicit calculation, in the following we use only the small $J^{P^{1}}=I^{P^{1}}$ for the base $S$. Hence from now on we let $t \in H^{2}(X)$ be a general divisor variable $t=t^{1} h+t^{2} \xi+t^{3} p$.

The hypergeometric factor is then explicitly given by

$$
I_{\beta}^{X}=I_{\beta}^{X / S} I_{\beta_{S}}^{S} \equiv I_{s, d}=\frac{\prod_{-d+s^{+}}^{0^{+}}\left(b_{0}+m z\right) \prod_{-d^{+}}^{0^{+}}\left(b_{1}+m z\right)}{\prod_{0^{+}}^{d-5 s^{+}}\left(a_{0}+m z\right) \prod_{0^{+}}^{d^{+}}\left(a_{1}+m z\right)} \times \frac{1}{\prod_{0^{+}}^{s^{+}}(p+m z)^{2}} .
$$

The product factor is positively directed in the sense that it needs to be switched up or down for smaller $d$ so that the running index $m$ is increasing. For example, if $s \in \mathbb{N}$ then $\prod_{0^{+}}^{s^{+}}$means $m \in\left[0^{+}, s^{+}\right] \cap \mathbb{Z}$, namely $m \in[\epsilon, s+\epsilon] \cap \mathbb{Z}=\{1,2, \cdots, s\}$. However if $s \in \mathbb{Z}_{\leq 0}$ then

$$
\prod_{0^{+}}^{s^{+}}=1 / \prod_{s^{+}}^{0^{+}}
$$

and $\left[s^{+}, 0^{+}\right] \cap \mathbb{Z}=\{s+1, \cdots,-1,0\}$. The position shown above is thus correct for $d \geq 5 s$. Notice also the product length is simply $\left(\beta . a_{i}\right)$ or $\left(\beta . b_{i}\right)$ etc..

If $d \geq s+1$, the $z$ expansion is

$$
I_{s, d}=z^{2 s-2} W_{s}(d) b_{0} b_{1}+\cdots,
$$

where we call $W_{s}(x)$ the fundamental rational function:

$$
W_{s}(x)=\frac{(-1)^{s}}{(s !)^{2}} \frac{(x-(s+1)) \cdots(x-(5 s-1))}{x}, \quad \operatorname{deg} W_{s}=4 s-2 .
$$


In general the poles of $W_{s}(d)$ must lie entirely in the $\mathscr{F}$-effective range. Here in the example we have only one pole at $d=0$.

When $d$ moves from regular point of $W_{s}(d)$ into poles, the $z$ power will increase. In fact, the following beautiful relation holds:

$$
\left.I_{s, d}\left(z, z^{-1}\right)\right|_{a_{i}=1, b_{i}=-1}=W_{s}(d+1 / z),
$$

the Laurent series at $x=d$ with the formal assignment $\Delta x=1 / z$. Here, for $s \geq 1$, the residue of $W_{s}(d)$ at 0 is given by $w_{s}=(5 s-1) ! /(s !)^{3}$. In fact

$$
I_{s, 0}=(-1)^{s-1} w_{s} z^{2 s-2} a_{0}\left(z-\left(b_{0} H_{s}+2 p H_{s}+a_{0} H_{5 s-1}\right)+O(1 / z)\right) .
$$

Let $s=1$. Since the highest $z$ power is given by the term $A=w_{1} a_{0} z$, the first step BF then reads as

$$
B_{1}(z) I=I-w_{1} \bar{q} z\left(z \partial_{a_{0}}\right) I=O\left(z^{0}\right) \bmod \bar{q}^{2} .
$$

Here again we write $\partial_{a_{0}}$ to denote the operator $\partial_{t^{1}}-5 \partial_{t^{3}}$. We will do so in all the remaining sections. By the symmetry of roots, the operator $B_{1}(z)=1-w_{1} \bar{q} z\left(z \partial_{a_{0}}\right)$ is easily seen to be $\mathscr{F}$-invariant.

For $s=0, d \geq 1$, again from the explicit expression of $I_{s, d}$ we have extremal parts

$$
q^{d} e^{\frac{t}{z}+(t . \beta)} I_{0, d}=q^{d} e^{\frac{t^{1} h+t^{2} \xi+t^{3} p}{z}+t^{1} d}\left(\frac{b_{0} b_{1}}{d^{2}} \frac{1}{z^{2}}+O\left(1 / z^{3}\right)\right) .
$$

The $q^{d} \bar{q} z^{0}$ coefficient of $B_{1}(z) I=I-w_{1} \bar{q} z\left(z \partial_{a_{0}}\right) I$ then equals

$$
b_{0} b_{1}\left(W_{1}(d)-\frac{w_{1}}{d}\right)=: P_{1}(d) b_{0} b_{1}, \quad(d \geq 2) .
$$

By comparing with the similar calculation on the $X^{\prime}$ side, we observes the following polynomial property

$$
P_{1}^{X}(d)=-P_{1}^{X^{\prime}}(-d) .
$$

Modulo some terms in the $\mathscr{F}$-effective range, this implies the $\mathscr{F}$-invariance of the top $z$ power series in $B_{1}(z) I$ since

$$
\mathscr{F} \sum_{d \geq 0} P_{1}^{X}(d) b_{0} b_{1} q^{d}-\sum_{d \geq 0} P_{1}^{X^{\prime}}(d) b_{0}^{\prime} b_{1}^{\prime} q^{\prime d}=b_{0}^{\prime} b_{1}^{\prime} P_{1}(\delta) E\left(q^{\prime}\right)=0 .
$$

(Notice that we had not yet treat terms with $0 \leq d \leq 1$ carefully.)

This in turn will imply the $\mathscr{F}$-invariance of the operator $B_{2}(z)$ defined by

$$
B_{2}(z) I=\left(1-w_{1} \bar{q} z z \partial_{a_{0}}-\bar{q} \sum_{d \geq 1} q^{d} P_{1}(d) \widehat{b_{0} b_{1}}+U\right) I=1+O(1 / z),
$$

where $U$ denote terms in the $\mathscr{F}$-effective range which can be determined by a direct calculation (see the next section).

And then this would finally (and still highly non-trivially) implies the $\mathscr{F}$ invariance of the $1 / z$ coefficient, i.e. the GMT:

$$
\mathscr{F} \tau^{X}(t) \cong \tau^{X^{\prime}}(\mathscr{F} t)
$$

for $t \in H^{2}(X)$. This is carried out in the following section. 


\section{Explicit GMT}

We start by writing out the complete form of BF including the $\mathscr{F}$-effective range. Let $\Theta=b_{0} b_{1}$. Notice that $\hat{\Theta}=\widehat{b_{0} b_{1}}=\hat{b}_{0} \hat{b}_{1}-\hat{a}_{0} \hat{a}_{1}$ instead of being $\hat{b}_{0} \hat{b}_{1}$. This is because the quantization of a class is defined only on its reduced representation. In fact

$$
\hat{\Theta}=(1-q) \hat{b}_{0} \hat{b}_{1}
$$

since $\hat{a}_{0} \hat{a}_{1}=q \hat{b}_{0} \hat{b}_{1}$ by the Picard-Fuchs equation. To distinguish these two expressions we set $\hat{\Theta}_{2}=\hat{b}_{0} \hat{b}_{1}$ in the following and thus $\hat{\Theta}=(1-q) \hat{\Theta}_{2}$.

Lemma 7.1. Modulo $\bar{q}^{2}$, we have Birkhoff factorization

$$
\begin{aligned}
& \tilde{I}-w_{1} \bar{q} z z \partial_{h-5 p} \tilde{I} \\
& +w_{1} \bar{q}\left(H_{4} z \partial_{h-5 p} z \partial_{h-5 p}+2 H_{1} z \partial_{h-5 p} z \partial_{p}+H_{1} z \partial_{h-5 p} z \partial_{\xi-h+p}\right) \tilde{I} \\
& +w_{1} \bar{q} q\left(H_{1}-H_{4}\right) \hat{\Theta}_{2} \tilde{I} \\
& +\bar{q} q W_{1}(1)\left(z \partial_{h-5 p} z \partial_{\xi-h}+\hat{\Theta}_{2}\right) \tilde{I} \\
& \quad-\bar{q}(1-q) \sum_{d \geq 1} P_{1}(d) q^{d} \hat{\Theta}_{2} \tilde{I} .
\end{aligned}
$$

Here $P_{s}(d)$ denotes the polynomial part of $W_{s}(d)$ and $H_{n}:=\sum_{k=1}^{n} 1 / k$.

As before, this is deduced from the basic $z$ expansions in various ranges. In the following we called $\mathscr{F}$-effective range the unstable range since the $I$ factors jumps up and down unstably. The initial unstable range is the subrange where $W_{s}$ admit poles. In the current example there is only one pole for $W_{s}$ at $d=0$. Other effective range is then called the stable range.

Lemma 7.2 (Stable range). Let $s \geq 0$ and $d \geq s+1$, then

$$
I_{s, d}=W_{s}(d) \frac{\Theta}{z^{2-2 s}}\left(1-\frac{1}{z}\left((-h+p) H_{d-s-1}+2 p H_{s}+(h-5 p) H_{d-5 s}+\frac{h}{d}\right)+O\left(z^{-2}\right)\right) .
$$

This includes the extremal case $s=0, d \geq 1$.

REMARK 7.3 (Extremal term). Let $d \geq 1$. Then

$$
I_{0, d}=\frac{1}{d^{2}} \frac{\Theta}{z^{2}}\left(1-\frac{1}{z}\left((-h+p) H_{d-1}+(h-5 p) H_{d}+\frac{h}{d}\right)+O\left(z^{-2}\right)\right) .
$$

Recall that $w_{s}:=(5 s-1) ! /(s !)^{3}$ is the degree -1 coefficient of the Laurent series of $W_{s}(d)$ at $d=0$.

Lemma 7.4 (Initial unstable range). Let $s \geq 1$ and $d=0$, then

$$
\begin{aligned}
I_{s, 0} & =(-1)^{s-1} w_{s} z^{2 s-1}(h-5 p) \\
& \left(1-\frac{1}{z}\left((\xi-h+p) H_{s}+2 p H_{s}+(h-5 p) H_{5 s-1}\right)+O\left(z^{-2}\right)\right) .
\end{aligned}
$$

Lemma 7.5 (Remaining unstable range). Let $s \geq 1$ and $1 \leq d \leq s$, then

$$
\begin{aligned}
I_{s, d} & =-W_{s}(d) \frac{(h-5 p)(\xi-h)}{z^{2-2 s}} \times \\
& \left(1-\frac{1}{z}\left((\xi-h+p) H_{s-d}+(\xi-h) H_{d-1}+2 p H_{s}+(h-5 p) H_{5 s-d-1}\right)+O\left(z^{-2}\right)\right) .
\end{aligned}
$$


Now we determine $I_{1}^{+}$, the $z^{-1}$ term after BF (and using $\Theta \xi=0$ and $(h-5 p) h=0$ for simplification). The GMT is given by $\tau=t+I_{1}^{+}$.

$$
\begin{aligned}
I_{1}^{+}=w_{1} \bar{q}(h-5 p)\left((\xi-h+p)^{2}+(h-5 p)(\xi-h+p) H_{4} H_{1}+2(\xi-h+p) p H_{1}^{2}\right) \\
\quad+\bar{q} q W_{1}(1)(h-5 p)(\xi-h)\left(2 p H_{1}+(h-5 p) H_{3}+h H_{1}\right) \\
\diamond-\bar{q} \Theta \sum_{d \geq 2} W_{1}(d) q^{d}\left((-h+p) H_{d-2}+(-h) H_{d-1}+2 p H_{1}+(h-5 p) H_{d-5}+h H_{d}\right) \\
\S-w_{1} \bar{q} \Theta\left((h-5 p) \sum_{d \geq 1} \frac{1}{d^{2}} q^{d}-\sum_{d \geq 1}\right. \\
\left.\quad \frac{1}{d} q^{d}\left((-h+p) H_{d-1}+(-h) H_{d-1}+(h-5 p) H_{d}+h H_{d}\right)\right) \\
\quad+w_{1} \bar{q} \Theta\left(2 H_{4}(h-5 p)+2 H_{1} p+H_{1}((-h+p)-(h-5 p))\right) \sum_{d \geq 1} \frac{1}{d} q^{d} \\
b-w_{1} \bar{q} \Theta\left(H_{4}-H_{1}\right) \sum_{d \geq 1} q^{d}\left((-h+p) H_{d-1}+(-h) H_{d-1}+(h-5 p) H_{d}+h H_{d}\right) \\
b-w_{1} q \bar{q} \Theta\left(H_{1}-H_{4}\right)((-h+p)+(-h)) \sum_{d \geq 1} \frac{1}{d} q^{d} \\
b-w_{1} \bar{q} q \Theta\left(H_{1}-H_{4}\right) \sum_{d \geq 1} q^{d}\left((-h+p) H_{d-1}+(-h) H_{d-1}+(h-5 p) H_{d}+h H_{d}\right) \\
\sharp+q \bar{q} \Theta W_{1}(1)((-h)-(h-5 p)-(-h)-(-h+p)) \sum_{d \geq 1} \frac{1}{d} q^{d} \\
\sharp+\bar{q} \Theta \sum_{d \geq 1} q^{d} P_{1}(d)(1-q)((-h)+(-h+p)) \sum_{d \geq 1} \frac{1}{d} q^{d} \\
\sharp+\bar{q} \Theta \sum_{d \geq 1} q^{d} P_{1}(d)(1-q) \sum_{d \geq 1} q^{d}\left((-h+p) H_{d-1}+(-h) H_{d-1}+(h-5 p) H_{d}+h H_{d}\right) .
\end{aligned}
$$

Here the terms labeled with $\diamond, \S, b$ and $\sharp$ are coming from the more degenerate terms (other than $q^{d} / d$ ) from $I,-w_{1} \bar{q} z z \partial_{h-5 p} I, w_{1} *$ and $W_{1}(d) *$ respectively.

The basic idea to arrange the terms is the following:

(1) The main terms should be the derivative $W_{1}^{\bullet}(d)$ from $\diamond$ since there are harmonic series $H_{d-2}-H_{d-5}$ coupled to $W_{1}(d)$.

(2) The next main term would be the remaining part from $\diamond$ which we will make it into $P_{1}(d)$ by subtracting suitable $w_{1} / d$.

(3) Other terms related to $P_{1}(d)$ are from $\sharp$, there we need to perform harmonic convolution like $P_{1}(d) *(1 / d)$ defined by

$$
(P * Q)(d)=\sum_{k=1}^{d-1} P(d-k) Q(k) .
$$

When $Q(d)=1 / d$, it follows easily from the definition that

$$
P(d) *(1 / d)=P(d) H_{d-1}+P^{*}(d)
$$

where $P^{*}$ is a polynomial in $d$ with $\operatorname{deg} P^{*}=\operatorname{deg} P$.

(4) All non-polynomial terms left are either $q^{d} / d$ or $q^{d} /(d-1)$. For the latter, terms in the group labeled with $b$ will cancel automatically. The same holds for the group $\sharp$. In fact this auto-cancelation should follow from the beginning if we replace 
$h^{2}$ by $5 h p$ before the quantization. The final cancelation on $q^{d} / d$ follows by direct calculation.

Now we give the details. We omit all the $\bar{q} \Theta$ factor involved. From $\diamond$ we get

$$
\sum_{d \geq 2} W_{1}(d) q^{d}\left((h-5 p)\left(H_{d-2}-H_{d-5}\right)-\frac{h}{d}-2 p+4 p H_{d-2}\right) .
$$

From $\S$, the first series reduces the term $(h-5 p) H_{d}$ into $(h-5 p) H_{d-1}$ and we get

$$
w_{1} \sum_{d \geq 1} \frac{q^{d}}{d}\left(-4 p H_{d-1}+\frac{h}{d}\right) .
$$

By adding the $d=1$ terms, these two combines into $(2 p+h) W_{1}(1)$ plus

$$
(h-5 p) \sum_{d \geq 1} W_{1}^{\bullet}(d)+\sum_{d \geq 1} q^{d}\left(W_{1}(d)\left(-2 p-\frac{5 p}{d}+4 p H_{d-2}\right)+\frac{w_{1}}{d}\left(\frac{h}{d}-4 p H_{d-1}\right)\right) .
$$

The second sum simplifies to

$$
\sum_{d \geq 1} q^{d} P_{1}(d)\left(-2 p-\frac{5 p}{d}+4 p H_{d-2}\right)+w_{1} \sum_{d \geq 1} q^{d}\left(\frac{h-5 p}{d^{2}}-\frac{2 p}{d}-\frac{4 p}{(d-1) d}\right) .
$$

The $1 / d^{2}$ term in the second sum cancels the only singularity of $W_{1}^{\bullet}(d)$. Also

$$
\frac{-4 p w_{1}}{(d-1) d}=-4 p w_{1}\left(\frac{1}{d-1}-\frac{1}{d}\right)
$$

We will first deal with $1 /(d-1)$ terms. The first $\sharp$ series gives $4 p W_{1}(1) /(d-1)$. The last series in $\sharp$ gives

$$
\sum_{d \geq 1} q^{d} P_{1}(d)(1-q) \sum_{d \geq 1} q^{d}\left(\frac{2 h-p}{d}-4 p H_{d}\right)
$$

The part on $(2 h-p) / d$ exactly cancels with the second series in $\sharp$. The remaining part $-4 p(1-q) \sum_{d \geq 1} H_{d}=-4 p \sum_{d \geq 1} 1 / d$. Hence we arrive at

$$
-4 p \sum_{d \geq 1} P_{1}(d) *(1 / d)=-4 p \sum_{d \geq 1}\left(P_{1}(d) H_{d-1}-\frac{3}{2} d(d-1)-9(d-1)\right) .
$$

Now the first term cancels with a similar term $P_{1}(d) 4 p H_{d-2}$ above to

$$
-4 p \frac{P_{1}(d)}{d-1}=-4 p \frac{-d^{2}+9 d-26}{d-1}=4 p(d-8)+4 p \frac{18}{d-1},
$$

where surely $18=-P_{1}(1)$ and in fact the actual numbers are not important at all.

In summary, the above $1 /(d-1)$ terms sum together to

$$
\frac{4 p}{d-1}\left(-w_{1}+W_{1}(1)-P_{1}(1)\right)=0 \text { ! }
$$

Similarly and much easily, for the $b$ terms, the first and the third series differ by a shifting factor $q$ hence they combine into $-w_{1}\left(H_{4}-H_{1}\right)$ times

$$
\sum_{d \geq 1}\left(\frac{-h+p}{d-1}+\frac{-h}{d-1}+\frac{h-5 p}{d}+\frac{h}{d}\right)=\sum_{d \geq 1}\left(\frac{-2 h+p}{d-1}+\frac{2 h-5 p}{d}\right) .
$$

It is clear the first term cancels exactly the second series in $b$. 
It remains to take care $\sum_{d \geq 1} q^{d} / d$ terms. The coefficients are simply

$$
\begin{aligned}
& w_{1}\left(2 H_{4}(h-5 p)+2 p-2 h+6 p\right) \\
& \quad-5 p P_{1}(0)-2 p w_{1}+4 p w_{1}-w_{1}\left(H_{4}-H_{1}\right)(2 h-5 p)=0,
\end{aligned}
$$

which follows by noticing that $-P_{1}(0)=w_{1}\left(H_{4}-H_{1}\right)$. (The first line comes from the unmarked series between $\S$ and $b$.)

Finally, It is easy to see the resulting polynomial series is given by

$$
\begin{aligned}
\bar{q} \Theta & \sum_{d \geq 1} q^{d}\left((h-5 p) P_{1}^{\bullet}(d)+6 p(d+6)(d-1)+4 p(d-8)\right) \\
& \left.=\bar{q} \Theta \sum_{d \geq 1} q^{d}\left((h-5 p) P_{1}^{\bullet}(d)-6 p P_{1}(d)+8(11 d-28)\right)\right) .
\end{aligned}
$$

As we have explained before, this polynomial property together with its counterpart on the $X^{\prime}$ side will then leads to the analytic continuation of the generalized mirror transformation.

\section{References}

1. J. Brown; Gromov-Witten invariants of toric fibrations. arXiv:0901.1290.

2. Y. Iwao, Y.-P. Lee, H.-W. Lin \& C.-L. Wang; Invariance of Gromov-Witten theory under a simple flop, to appear in J. Reine. Angew. Math., arXiv:0804.3816.

3. Y.-P. Lee, H.-W. Lin \& C.-L. Wang; Flops, motives and invariance of quantum rings, Annals of Math., 172 (2010), no.1, 243-290.

4. Y.-P. Lee, H.-W. Lin \& C.-L. Wang; Invariance of quantum rings under ordinary flops, arXiv:1109.5540.

5. A.-M. Li \& Y. Ruan; Symplectic surgery and Gromov-Witten invariants of Calabi-Yau 3-folds, Invent. Math. 145 (2001), 151-218.

6. C.-L. Wang; On the topology of birational minimal models, J. Diff. Geom. 50 (1998), 129-146.

Department of Mathematics, University of Utah, Salt Lake City, Utah

E-mail address: yplee@math.utah.edu

Department of Mathematics and Taida Institute of Mathematical Sciences (Tims), National Taiwan University, TAipei, TAiwan

E-mail address: linhw@math.ntu.edu.tw

Department of Mathematics, Center for Advanced Studies in Theoretic Sciences (CASTS) and Taida Institute of Mathematical Sciences (TIMS), National Taiwan UniVERSITY, TAIPEI, TAIWAN

E-mail address: dragon@math.ntu.edu.tw 